\title{
A Sound Source Location Method Based on Wireless Sensor Network
}

\author{
Jiang Ming Liu Xiaowei Sang Shengtian Wang Sida \\ MEMS Center, Harbin Institute of Technology, Harbin, Heilongjiang, China
}

\begin{abstract}
The sound source location method includes time delay estimation and location. This paper proposes a time delay estimation algorithm based on the arc tangent transformation and the algorithm still has a good stability when the background noise of the public places is non-Gaussian noise. Secondly, the range and angle location algorithm was used to determine the location of sound source and the error analysis was carried out. The research results show that the cross shaped array with five elemnets has good loaction performance because it can eliminate the effect of location error caused by azimuth angle.
\end{abstract}

Keywords: sound source localization; time delay estimation; wireless sensor network

\section{Introduction}

The noise pollution is an important part of the modern environmental pollution, therefore, the environmental noise monitoring has become really important as a means of the evaluation on the environment. The conventional noise monitoring transmits the collected data by wired medium that causes a few shortcomings, such as the difficulties of cable casting and the high cost. Thus the current research focuses on the noise monitoring based on wireless sensor network because of the smaller cost, the easier cabling, the higher precision and the more powerful synergy ${ }^{[1]}$. Wireless sensor network is a new technique and achieves more attractions in a lot of countries $^{[2 \sim 4]}$. Location of the noise source is a key technology in the noise monitoring. In this paper, a sound source location method based on wireless sensor network was proposed and an improved time delay estimation method based on the arc tangent transformation and its perfromances were simulated.

\section{Time Delay Estimation Algorithm}

The sound wave propagates in the air in the form of spherical wave. The speed of sound is determined. The arriving paths of sound signal from the sound source to two sensors are different, so the time delay is produced for these two sensors. The range difference from the wave front of the sound signal to two sensors is called as sound path difference. Assume that $L$ is the distance between two sound sensors, $D$ is the time delay, $c$ is the sound speed, $H$ is the sound path difference, $\theta$ is the direction angle between sound source and sensors, which can determine the location of sound source. An ideal mathematical model can be written as follows:

$$
\left\{\begin{array}{c}
x_{1}(t)=s(t)+w_{1}(t) \\
x_{2}(t)=\alpha s(t-D)+w_{2}(t)
\end{array}\right.
$$

Where $x_{1}(t)$ and $x_{2}(t)$ are the received sound signals of two sensors, respectively, $s(t)$ is the sound source signal, $w_{1}(t)$ and $w_{2}(t)$ are the received noise signals of two sensors, $D$ is the time delay between 
two sensors, $\alpha$ is the attenuation coefficient. Here assume that $w_{1}(t)$ and $w_{2}(t)$ are the uncorrelated steady noise, further they are not related to $s(t)$. For the discrete time system, the model can be expressed as:

$$
\left\{\begin{array}{c}
x_{1}(n)=s(n)+w_{1}(n) \\
x_{2}(n)=\alpha s(n-D)+w_{2}(n)
\end{array}\right.
$$

From above, we can see that the time delay can be estimated based on the sound source signals of two sensors. The common sound source estimation algorithm includes Generate Cross Correlation (GCC) and Least Mean Square (LMS). The algorithm flow diagram of GCC is shown in Fig.1.

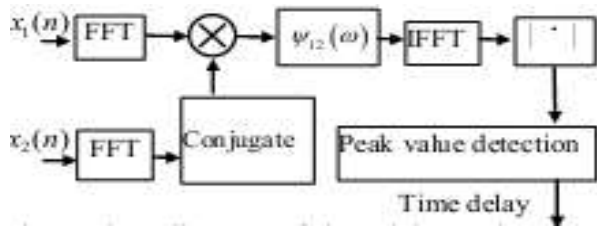

Fig.1: Flow diagram of time delay estimation based on GCC.

The expression of GCC is:

$$
R_{x_{1} x_{2}}(\tau)=\int_{0}^{\pi} \psi_{12}(\omega) G_{x_{1} x_{2}}(\omega) e^{-j \omega \tau} d \omega
$$

Where $\psi_{12}(\omega)$ is the weighting function and $G_{x_{1} x_{2}}(\omega)$ is the cross correlation power spectrum density function which can be expressed as follows.

$$
G_{x_{1} x_{2}}(\omega)=X_{1}(\omega) X_{2}^{*}(\omega)
$$

$X_{1}(\omega)$ and $X_{2}(\omega)$ are the Fourier transformations of $x_{1}(\mathrm{n})$ and $x_{2}(\mathrm{n})$, respectively. When the weight function is $1 /\left|G_{x_{1} x_{2}}(\omega)\right|$, it is called as PHAT weighting and the algorithm is called as GCC-PHAT.

In public places, background noises are not Gauss noise, so the $\alpha$ steady distribution model was introduced and its eigen function can be written as:

$$
\varphi(t)=\exp \left\{j a t-\gamma|t|^{\alpha}[1+j \beta \operatorname{sgn}(t) \omega(t, \alpha)]\right\}
$$

Where $\operatorname{sgn}(\cdot)$ is the symbol function and it can be expressed as:

$$
\operatorname{sgn}(t)= \begin{cases}1, & t>0 \\ 0, & t=0 \\ -1, & t<0\end{cases}
$$

And $\omega(t, \alpha)$ is:

$$
\omega(t, \alpha)= \begin{cases}\tan \frac{\alpha \pi}{2}, & \alpha \neq 1 \\ \frac{2}{\pi} \log |t|, & \alpha=1\end{cases}
$$

Here $\alpha, \beta, \gamma$ and $a$ are the four parameters of $\alpha$ steady distribution. When $\beta=0, \quad \alpha$ steady distributions called as symmetrical $\alpha$ steady distribution or $S \alpha S$.

In this section, an improved PHATGCC time delay estimation method based on the arc tangent transformation was presented, which is called as ATANPHAT algorithm. The nonlinear transformations of $x_{1}(n)$ and $x_{2}(n)$ are based on the tangent function, namely:

$$
\begin{aligned}
& r_{1}(n)=\arctan \left[k x_{1}(n)\right] \\
& r_{2}(n)=\arctan \left[k x_{2}(n)\right]
\end{aligned}
$$

Where $k$ is the parameter which is used to control the transformation degree.

The arc tangent function is the limited function, when the angle is small, the arc tangent function is close to linear transformation and it not affects signals seriously. When the large pulse appears, the arc tangent function maps the input signal into $(-\pi / 2, \pi / 2)$,so the pulse signal can be suppressed.

In order to improve the performance of PHAT-GCC in the case of low Ratio of Signal to Noise (SNR) and reverberation environment, the weighting function was changed to: 


$$
\psi_{12}^{\prime}=1 /\left|G_{x_{1} x_{2}}(\omega)\right|^{\lambda}
$$

Where $0.5 \leq \lambda \leq 1$ and it changes with the SNR:

$$
\lambda=\left\{\begin{array}{lr}
\lambda_{0}, & \sigma<\sigma_{0} \\
\frac{\lambda_{1}-\lambda_{0}}{\sigma_{1}-\sigma_{0}}\left(\sigma-\sigma_{1}\right)+\lambda_{1}, & \sigma_{0} \leq \sigma \leq \sigma_{1} \\
\lambda_{1}, & \sigma<\sigma_{1}
\end{array}\right.
$$

Here $\sigma$ is $\mathrm{SNR}, \sigma_{0}$ and $\sigma_{1}$ are the upper bound and lower bound for the actual case, $\lambda_{0}$ and $\lambda_{2}$ are constants determined according to the actual case. After the weighting function is introduced, the cross power spectrum function can be written as:

$$
G_{x_{1} x_{2}}^{\prime}(\omega)=\psi_{12}^{\prime}(\omega) G_{x_{1} x_{2}}(\omega)
$$

In order to sharpen the peak value of cross correlation function, power spectrum density, the accumulation weighting is used to the cross power spectrum function and the cross power spectrum function after accumulation weighting is:

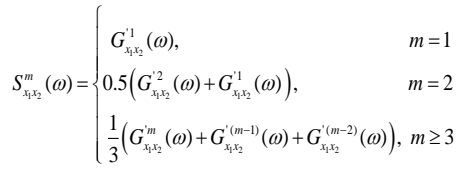

Where $m$ is the frame number and $G_{x_{1} x_{2}}^{\prime m}(\omega)$ is the cross correlation function of the $m$ th frame. The cross correlation function can be obtained through the formula (14).

$$
R_{x_{1} x_{2}}^{m}(\tau)=\int_{0}^{\pi} S_{x_{1} x_{2}}^{m}(\omega) e^{-j \omega \tau} d \omega
$$

The peak value of the above function is the time delay of two sound sensors.

This paper simulated the performances of proposed algorithm for variant SNR.

The background noise was subject to fractional and low order $\alpha$ steady distribution. $\alpha$ is designated to be 1.8 .
The concept of Generation Signal of Noise Ratio (GSNR) was introduced, which can be expressed as:

$$
G N S R_{d B}=10 \lg \left(\sigma_{s}^{2} / \gamma\right)
$$

Where $\sigma_{s}^{2}$ is the variance of sound source signal, $\gamma$ is the dispersion coefficient FLOA distribution noise. The simulation results were shown in Fig.2.

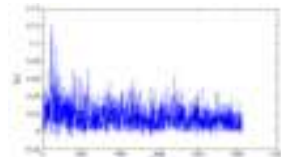

(a) GCC-PHAT for $\mathrm{GSNR}=10 \mathrm{~dB}$

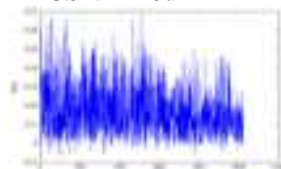

$$
\text { (c)GCC-PHAT for }
$$

GSNR $=0 \mathrm{~dB}$

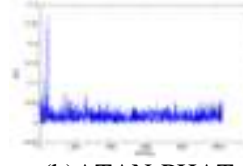

(b)ATAN-PHAT for $\mathrm{GSNR}=10 \mathrm{~dB}$

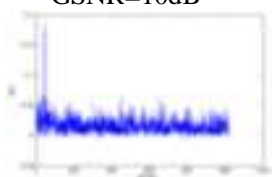

(d)ATAN-PHAT for $\mathrm{GSNR}=0 \mathrm{~dB}$
Fig.2: Simulation results of time delay estimation algorithms.

From Fig.2, we can see that the results of both GCC-PHAT and ATAN-PHAT have sharp peak values when $\mathrm{GSNR}=10 \mathrm{~dB}$. The correct time delay can be obtained according to these two methods in this case. When GSNR=0dB, GCC-PHAT has lost the ability of time delay estimation, but the cross correlation function waveform of ATAN-PHAT is still good and the amplitude of the peak value is still enough sharp, so its ability of time delay estimation is good.

\section{Location Method Based on Time Delay Estimation}

A five element sensor array is introduced which is shown in Fig.3. The distance between two elements is $d$, the coordinates of five nodes are $M_{0}(0,0,0)$, $M_{1}(d / 2,0,0), M_{2}(0, d / 2,0), M_{3}(-d / 2,0,0)$ and $M_{4}(0,-d / 2,0)$. The time delays of the arrived signals of $M_{1}, M_{2}, M_{3}$ and $M_{4}$ relative to $M_{0}$ are $\tau_{1}, \tau_{2}, \tau_{3}$ and $\tau_{4}$. The 
sound speed is $c$. The coordinates of sound source are:

$$
\begin{aligned}
& r=\left(d^{2}-c^{2} \sum_{i=1}^{4} \tau_{i}^{2}\right) /\left(2 c \sum_{i=1}^{4} \tau_{i}\right) \\
& \varphi=\arctan \left(\left(\tau_{2}-\tau_{4}\right) /\left(\tau_{1}-\tau_{3}\right)\right) \\
& \theta \approx \arcsin \left(((2 c) / d) \sqrt{\left(\tau_{1}-\tau_{3}\right)^{2}+\left(\tau_{2}-\tau_{4}\right)^{2}}\right)
\end{aligned}
$$

Assume that the variances of time delay estimation are all $\sigma_{r}$, the variances of $\theta, \varphi$ and $r$ are:

$$
\begin{aligned}
& \sigma_{\varphi \tau}=\sqrt{\left(\frac{\partial \varphi}{\partial \tau_{1}} \sigma_{\tau}\right)^{2}+\left(\frac{\partial \varphi}{\partial \tau_{2}} \sigma_{\tau}\right)^{2}+\left(\frac{\partial \varphi}{\partial \tau_{3}} \sigma_{\tau}\right)^{2}+\left(\frac{\partial \varphi}{\partial \tau_{4}} \sigma_{\tau}\right)^{2}} \\
& \sigma_{\theta \tau}=\sqrt{\left(\frac{\partial \theta}{\partial \tau_{1}} \sigma_{\tau}\right)^{2}+\left(\frac{\partial \theta}{\partial \tau_{2}} \sigma_{\tau}\right)^{2}+\left(\frac{\partial \theta}{\partial \tau_{3}} \sigma_{\tau}\right)^{2}+\left(\frac{\partial \theta}{\partial \tau_{4}} \sigma_{\tau}\right)^{2}} \\
& \sigma_{r \tau}=\sqrt{\left(\frac{\partial r}{\partial \tau_{1}}\right)^{2} \sigma_{\tau}^{2}+\left(\frac{\partial r}{\partial \tau_{2}}\right)^{2} \sigma_{\tau}^{2}+\left(\frac{\partial r}{\partial \tau_{3}}\right)^{2} \sigma_{\tau}^{2}+\left(\frac{\partial r}{\partial \tau_{4}}\right)^{2} \sigma_{\tau}^{2}} \\
& \sigma_{\varphi \tau} \approx \frac{2 \sqrt{2} c}{d \sin \theta} \sigma_{\tau} \\
& \sigma_{\theta \tau} \approx \frac{4 \sqrt{2} c}{d \cos \theta} \sigma_{\tau} \\
& \sigma_{r \tau} \approx \frac{4 r c \sqrt{\left(\frac{d}{2}\right)^{2}+r^{2}}}{\left(\frac{d}{2}\right)^{2}\left(4-\sin ^{2} \theta\right)} \sigma_{\tau}
\end{aligned}
$$

It can be seen that the errors of $\theta$ and $\varphi$ are not relative to $\varphi$, which are relative to $c, d$ and $\theta$, so one error factor is reduced for the direction location of sound source for five element array. The location precision is not relative to the azimuth direction, which can notably improve the location precision at the azimuth direction.

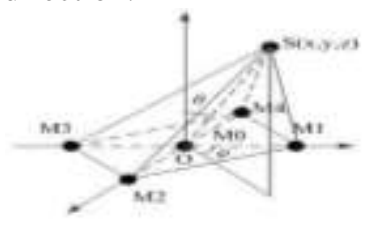

Fig.3: Five element sensor array.

\section{Conclusion}

The method proposed in this paper includes an improved PHAT-GCC time delay estimation algorithm and location algorithm based on it. The simulation results indicate that the improved time delay estimation algorithm is more suitable for public places with non-Gauss noise than traditional algorithm. At the same time, the research results reveal that the cross shaped array with five elemnets has better loaction performance than the cross shaped array with four elemnets because it can eliminate the effect of location error caused by azimuth angle. The research results in this paper can provide a new sound source location method for practical applications.

\section{Acknowledgement}

The authors would like to acknowledge the financial support from the national high technology research and development program of china (Grant No. SS 2012AA062902), Major Science and Technology Program for Water Pollution Control and Treatment (Grant No. 2012ZX07201003-01), Key Science and Technology Project of Harbin (Grant No. 2011AA1CG003).

\section{References}

[1] Arici T, Altunbasak Y, "Adaptive Sensing for Environment Monitoring Using Wireless Sensor Networks", IEEE Wireless Communications and Networking Conference, pp. 23472352, 2004.

[2] Culler D, Estrin D, Srivastava M, "Overview of Sensor Newtorks", IEEE Computer Society, pp. 41-49, 2004.

[3] Bult K, Burstein A, Chang D, "Wireless Integrated Microsensors", Solid-State Sensor and Actuator Workshop, pp. 205-210, 1996.

[4] Stankovic J A, Lee I, Mok A, "Opportunities and Obligations for Physical Computing Systems", IEEE Computer Society, pp. 23-31, 2005. 